\title{
Análisis del concepto de sostenibilidad y su aplicación en las políticas de turismo en Colombia*
}

\author{
Adriana Bermúdez Cardona** \\ Carlos Alberto Zárate Yepes ${ }^{* \star *}$
}

Recibido: 3 de septiembre de 2016

Enviado a pares evaluadores: 12 de septiembre de 2016 Aprobado por pares evaluadores: 27 de octubre de 2016 Aprobado por comité editorial: 10 de noviembre de 2016 DOI: $10.22395 /$ csye.v5n10a6

\section{RESUMEN}

La actividad turística siempre se ha destacado por generar un desarrollo en diferentes sectores económicos. Su principal materia prima es básica: puede ir desde un simple atractivo natural, una infraestructura turística, una fiesta o hasta un plato típico. Sin embargo, todavía esta actividad económica cuyo principal insumo es el servicio, y en donde no se fabrican productos, sigue desarrollándose en muchos casos de manera informal y mal planificada, generando un deterioro de los diferentes recursos naturales y culturales.
Es por todo eso que Colombia viene hoy formulando políticas orientadas al ordenamiento y regulación de esta actividad con un enfoque sostenible; sin embargo el reconocimiento y aplicación por parte del sector no ha sido claro debido a la forma como desde la política se concibe la sostenibilidad y, por ende, el turismo sostenible. Este trabajo hace un recorrido crítico por la forma como la sostenibilidad se incorpora y aplica en la legislación turística.

Palabras clave: Leyes y políticas de turismo; turismo sostenible; sostenibilidad; desarrollo sostenible.

Este artículo muestra parte de los resultados obtenidos dentro del trabajo de investigación de la Maestría en Ciencias Ambientales, donde desde el principio se partió de la inquietud de cómo las políticas de turismo en Colombia abordaban el tema de la sostenibilidad, por lo cual se hizo un barrido por toda la normativa de Colombia y los momentos más importantes al nivel mundial donde el tema de la sostenibilidad había sido abordado, lo que dio como resultado la confirmación de la poca articulación de las políticas con la sostenibilidad y resaltó la necesidad de que se diseñe una política puntual que trabaje la sostenibilidad en turismo en Colombia.

** Administradora de Empresas Turísticas del Colegio Mayor de Antioquia. Candidata a magíster en Ciencias Ambientales de la Universidad de Antioquia. Coordinadora de los programas de Turismo en la Corporación Académica Ambiental y docente de cátedra. Correo electrónico: adriana.bermudez@udea.edu.co

*** Abogado de la Universidad de Antioquia, economista agrícola de la Universidad Nacional de Colombia, especialista y magíster en Derecho Procesal de la Universalidad de Medellín. Profesor asociado de la Universidad Nacional de Colombia, sede Medellín. Director del Grupo de Investigación Polygesta -Política, Legislación y Gestión Ambiental-, adscrito a la Facultad de Minas de la Universidad Nacional de Colombia, sede Medellín. Correo electrónico: cazarate@unal.edu.co 


\section{Analysis of the concept of sustainability and its application in the politics of tourism in Colombia}

\section{ABSTRACT}

The touristic activity always has stood by generating development in different economic sectors. Its main raw material is basic: it can go from a simple natural attraction, a tourist infrastructure, a party or, till a dish. However, this economic activity whose main asset is service and in which there aren't manufactured products, still is developing in many cases in an informal way and poorly planned, generating a deterioration of natural and cultural resources. This is why Colombia is currently formulating policies aimed at

planning and regulation of this activity with a sustainable approach. However, the recognition and the enforcement of the sector have not been clear due to the way that the policy conceives sustainability hence, sustainable tourism. This work makes a critical tour through the manner how sustainability is embodied and applied in the tourist legislation.

Keywords: tourism policies and laws; sustainable tourism; sustainability; sustainable development. 


\section{Introducción}

El turismo genera beneficios y efectos positivos en el contexto del desarrollo sostenible, pero al mismo tiempo representa un desafío para autoridades, empresarios, educadores, estudiantes y para todos los que son parte de esta actividad.

En el ámbito turístico y ambiental Colombia ha diseñado una amplia gama de instrumentos normativos que permiten orientar diversas actividades; de allí la importancia de realizar un análisis crítico de las políticas existentes de turismo sostenible para identificar cómo el concepto de sostenibilidad se ha concebido, y así poder contribuir a la discusión en procura de plantear una ruta que contribuya a un turismo donde se beneficien los actores de la cadena (sector público, privado, comunidades receptoras y turistas), así como los recursos que soportan dicha actividad.

Para el análisis del concepto de sostenibilidad en las políticas de turismo en Colombia se llevó a cabo el siguiente proceso:

1. El turismo en el marco del desarrollo sostenible. Un recuento de las actividades más relevantes que han marcado el tema de la sostenibilidad al nivel mundial y su relación con el turismo.

2. Análisis del concepto de sostenibilidad en los documentos normativos de turismo. Se seleccionaron las leyes, Conpes, políticas de turismo, normas técnicas sectoriales y planes sectoriales de turismo, se identificó el concepto de sostenibilidad y se hizo un análisis en cada caso.

3. Una mirada al desarrollo del turismo sostenible en Colombia. Se analizaron aspectos que han sido relevantes en el desarrollo del turismo sostenible en Colombia, para hacer una lectura crítica de los mismos.

\section{El turismo en el marco del desarrollo sostenible}

El desarrollo sostenible se entiende como un modelo de crecimiento "que satisfaga las necesidades de la presente generación sin comprometer la capacidad de las generaciones futuras para satisfacer sus propias necesidades" (Informe de la Comisión Mundial sobre Medio Ambiente y Desarrollo, 1987) ${ }^{1}$. Como lo destaca Raúl Brañes ${ }^{2}$ (Brañes, 2000), esta es la idea básica que postula el Informe de la Comisión Mundial sobre el Medio Ambiente y el Desarrollo (conocido

\footnotetext{
Cfr. Informe de la Comisión Mundial sobre el Medio Ambiente y el Desarrollo, Madrid, Alianza, 1987, p. 46.

2 Bañes, Raúl. "El acceso a la justicia ambiental en América Latina: Derecho ambiental y desarrollo sostenible", ponencia publicada en Derecho Ambiental y Desarrollo Sostenible. El acceso a la justicia ambiental en América Latina, Memoria del Simposio Judicial realizado en la ciudad de México del 26 al 28 de enero de 2000. Publicación de la Oficina Regional para América Latina y el Caribe y de la Procuraduría Federal de Protección al Ambiente, pp. 41 y 42.
} 
como el Informe Brundtland ${ }^{3}$ ) creada por las Naciones Unidas para impulsar su programa de cambio global. Este informe llevó a las Naciones Unidas a convocar a una conferencia sobre el Medio Ambiente y Desarrollo llamada "Cumbre de la Tierra ${ }^{4}$ ". En esta conferencia se aprobó una importante declaración y un plan de acción al nivel mundial que es conocido como la Agenda $21^{5}$.

Los problemas ambientales fueron una preocupación mundial principalmente desde los primeros años del siglo XX. En 1972, la Organización de Naciones Unidas convocó una conferencia sobre el Medio Ambiente que tuvo lugar en Estocolmo, la cual puso los temas ambientales en la agenda mundial y en la de todos los países del mundo, incluidos los de nuestra región. A partir de la Conferencia de Estocolmo, se fortaleció la planeación y la gestión ambiental, reforzando lo que ya existía en muchos países del mundo. Lo expresado implicó cambios en las políticas públicas y en los propios sistemas jurídicos, dentro de los cuales se insertaron normas con el claro propósito de proteger el medio ambiente y promover el desarrollo sostenible.

En Colombia el concepto de desarrollo sostenible se entiende como: "aquel que conduce al crecimiento económico, al mejoramiento de la calidad de vida y al bienestar social, sin agotar la base de recursos naturales renovables en que se sustenta, ni deteriorar el medio ambiente o el derecho de las generaciones futuras a utilizarlo para la satisfacción de sus propias necesidades", (artículo 3 de la Ley 99 de 1993). Este concepto implica una nueva forma de concebir las relaciones del ser humano frente a la naturaleza y es un avance para la consolidación de un modelo de crecimiento sostenido y sostenible.

En agosto del 2004, la OMT divulga, en el documento Desarrollo Sostenible del Turismo, una nueva definición sobre el mismo, enfatizando en el equilibrio que debe existir entre los aspectos ambientales, sociales y económicos del turismo, así como en la necesidad de aplicar principios de sostenibilidad en sus sectores, haciendo referencia, por ejemplo, a objetivos mundiales relacionados con la eliminación de la pobreza.

3 El libro Nuestro futuro común (nombre original del Informe Brundtland) fue el primer intento de eliminar la confrontación entre desarrollo y sostenibilidad. Presentado en 1987 por la Comisión Mundial para el Medio Ambiente y el Desarrollo de la ONU, encabezada por la doctora noruega Gro Harlem Brundtland.

4 Celebrada en Río de Janeiro, Brasil, los días 3 al 14 de junio de 1992, fue un momento decisivo en las negociaciones internacionales sobre las cuestiones del medio ambiente y el desarrollo. Con la participación de 173 Estados, y la presencia de 118 jefes de Estado y de Gobierno, aprobaron tres grandes acuerdos que habrían de regir la labor futura: el Programa 21, un plan de acción mundial para promover el desarrollo sostenible; la Declaración de Río sobre el Medio Ambiente y el Desarrollo, un conjunto de principios en los que se definían los derechos civiles y obligaciones de los Estados, y una Declaración de principios relativos a los bosques, directrices para la ordenación más sostenible de los bosques en el mundo.

5 Declara que la humanidad está en un momento crítico de la historia, que ninguna nación la puede enfrentar aisladamente y llama a una nueva era de cooperación internacional. Una impresionante convocatoria de la sociedad civil reunió, paralelamente a la Cumbre, a más de 20000 líderes sociales y ambientalistas. 
[...] las directrices para el desarrollo sostenible del turismo y las prácticas sostenibles son aplicables a todas las formas de turismo en todos los destinos, incluidos el turismo de masas y los diversos segmentos turísticos. Los principios de sostenibilidad se refieren a los aspectos ambiental, económico y sociocultural del desarrollo turístico, habiéndose de establecer un equilibrio adecuado entre estas tres dimensiones para garantizar su sostenibilidad a largo plazo (Acerenza, 2011).

Según: la Federación de Parques Nacionales y Naturales, el turismo sostenible lo constituyen: "todas las formas de desarrollo turístico, gestión, y actividad que mantienen la integridad ambiental, social y económica, así como el bienestar de los recursos naturales y culturales a perpetuidad" (FNNP, 1993).

Se resalta de la definición de la OMT, que deben existir directrices en el desarrollo del turismo sostenible debido a que, partiendo de una buena gobernabilidad y una adecuada planificación, se podrá garantizar que el proceso que se desarrolle estará más orientado a la sostenibilidad que otro que no cuente con estos elementos.

De todo este proceso hacia el turismo sostenible cabe resaltar los encuentros que se derivan de la Agenda 21 en la Cumbre de la Tierra, la construcción de un Código Mundial para el Turismo, encuentro realizado en Chile en el año de 1999; la Cumbre Mundial de Ecoturismo en Quebec (Canadá) en el año 2002 y de la cual sale una declaración que es socializada el mismo año en la Cumbre Mundial para el Desarrollo Sostenible en Johannesburgo (África).

Cabe anotar que dentro de la Cumbre de la Tierra en el año de 1992 no se trata directamente un capítulo sobre el turismo sostenible, pero fue un insumo clave para que la OMT y el Consejo Mundial de Viajes y Turismo elaborara un programa que diera cuenta del desarrollo del sector desde la sostenibilidad concebido como

[...] un desarrollo sostenible que atiende a las necesidades de los turistas actuales y de las regiones receptoras y al mismo tiempo protege y fomenta las oportunidades para el futuro. Se concibe como una vía hacia la gestión de todos los recursos de forma que puedan satisfacerse las necesidades económicas, sociales y estéticas, respetando al mismo tiempo la integridad cultural, los procesos ecológicos esenciales, la diversidad biológica y los sistemas que contienen la vida.

Todo este panorama nutre, a su vez, la Conferencia Mundial de Turismo Sostenible realizada en Lanzarote, España, en 1995 y de la cual emana la Carta de Turismo Sostenible sustentada en 18 principios. En esta se plantean entre otros temas vitales: la participación de las comunidades, la protección del patrimonio cultural y natural, el compromiso de las organizaciones, la responsabilidad de los entes gubernamentales, la elaboración de políticas y la importancia de la planificación en los territorios, la adopción y puesta en práctica de códigos de conducta que puedan implementarse en toda la cadena de valor de turismo. 
No es gratuito que en este contexto surja en el año de 1999 el Código de Ética Mundial para el Turismo como resultado de la Asamblea General de la Organización Mundial del Turismo.

El año 2002 es crucial para la cristalización de la sostenibilidad en el turismo: se declara dicho año como el Año Mundial del Ecoturismo, se realiza la Cumbre Mundial de Ecoturismo en Quebec (Canadá). Dicho trabajo centró las temáticas de la sostenibilidad en dos grandes temas transversales: el ecoturismo concebido desde lo ambiental, económico y sociocultural, y la participación y atribución de competencias a las comunidades locales y a los pueblos autóctonos en el procesos de desarrollo del ecoturismo, en la gestión y supervisión de las actividades ecoturísticas y en la distribución de los beneficios que reporten.

La declaración de Quebec sirve de base para la Cumbre Mundial de Desarrollo Sostenible en Johannesburgo y lo emanado de esta en cuanto al turismo sostenible da pie para que en el año 2004 se examine el concepto de sostenibilidad en el turismo, adaptándose así el siguiente texto:

[...] Las directrices para el desarrollo sostenible del turismo son aplicables a todas las formas del turismo en todos los destinos, incluidos el turismo de masas y los diversos segmentos turísticos. Los principios de sostenibilidad se refieren a los aspectos ambiental, económico y sociocultural del desarrollo turístico, habiéndose de establecer un equilibrio adecuado entre estas tres dimensiones para garantizar su sostenibilidad a largo plazo.

En el año 2010 se celebra la trigésimo cuarta reunión del Comité de la Organización Mundial del Turismo para Oriente Medio, realizada en Sana'a (Yemen). En dicho evento se organizó un Taller Internacional relativo al Informe sobre la economía verde en la Casa Internacional del Medio Ambiente, en Ginebra (Suiza). Las Naciones Unidas declararon este como año Internacional de la Diversidad Biológica y se creó, además, la Asociación Mundial para el Turismo Sostenible.

Una lectura al concepto de sostenibilidad en los documentos normativos de turismo en Colombia

No existe una política clara de turismo sostenible, debido a que la sostenibilidad en sí misma cuestiona el modelo economicista de desarrollo que impera en el país. Esta es quizá una de las razones por las cuales el tema de la sostenibilidad se aborda de manera muy tímida en los documentos asociados a planes y políticas turísticas. Se abordarán en esta lectura crítica las leyes, las políticas y los documentos Conpes donde figura el concepto de sostenibilidad (ver figura 1).

Colombia ha expedido tres leyes de turismo: Ley 300 de 1996 como rectora y dos leyes más modificatorias, la Ley 1101 de 2006 y la 1558 de 2012. Para 
nuestra reflexión se tendrá en cuenta la 300 y la 1558, ya que la 1101 se centra fundamentalmente en el tema de los parafiscales para el sector.

\begin{tabular}{|c|c|c|c|}
\hline \multirow{2}{*}{ 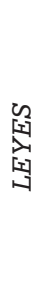 } & Ley 300/96 & \multicolumn{2}{|c|}{$\begin{array}{l}\text { Principio (5): Protección al ambiente: en virtud del cual el turismo se desarrollará en armonía con el } \\
\text { desarrollo sustentable del medio ambiente. } \\
\text { Artículo (16): [... El plan sectorial de turismo contendrá elementos para fortalecer la competitividad } \\
\text { del sector...] }\end{array}$} \\
\hline & Ley 1558/12 & \multicolumn{2}{|c|}{$\begin{array}{l}\text { Principio (9): Desarrollo sostenible: el turismo se desarrolla en armonía con los recursos naturales y } \\
\text { culturales, a fin de garantizar sus beneficios a las futuras generaciones, la determinación de la capacidad } \\
\text { de carga constituye un elemento fundamental de la aplicación de este principio. } \\
\text { Artículo (5): calidad turística }\end{array}$} \\
\hline \multirow{7}{*}{ 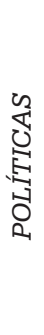 } & \multicolumn{2}{|c|}{ Política de Ecoturismo } & Principio y objetivo general \\
\hline & \multicolumn{2}{|c|}{ Política de Turismo Social } & Lineamientos \\
\hline & \multicolumn{2}{|c|}{ Política de Turismo Cultural } & Objetivos específicos, principios y lineamientos \\
\hline & \multicolumn{2}{|c|}{ Política de Turismo y Artesanías } & Principios, objetivos y lineamientos \\
\hline & \multicolumn{2}{|c|}{ Política de Playas Turísticas } & Principios, ejes temáticos, objetivo general y específicos, lineamientos \\
\hline & \multicolumn{2}{|c|}{ Política de Desarrollo Comunitario } & Principio, objetivo general y en los lineamientos \\
\hline & \multicolumn{2}{|c|}{ Política de Turismo de Naturaleza } & Princpios, ejes temáticos, objetivo general y lineamientos \\
\hline \multirow{3}{*}{ 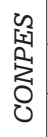 } & CONPES 3296 & \multicolumn{2}{|l|}{ Principios (3) y (4) } \\
\hline & CONPES 3658 & \multicolumn{2}{|c|}{ Objetivos (2) y Recomendaciones } \\
\hline & CONPES 3397 & \multicolumn{2}{|c|}{ Estrategias competitivas } \\
\hline
\end{tabular}

Figura 1. Recuento políticas de turismo en Colombia, con inclusión del concepto sostenible (adaptación personal)

En el año 2012 se crea la Ley 1558 que modifica la Ley 300 de 1996 y la 1101 de 2006. Dicha ley crea en su artículo 7 el Consejo Superior del Turismo, bajo la dirección del Ministerio de Comercio, Industria y Turismo, como órgano de coordinación entre los entes estatales con el propósito de armonizar el ejercicio de sus competencias con la política turística dictada por dicho Ministerio. Bajo esta misma ley se crea también el Consejo Consultivo de la Industria Turística, artículo 8, como órgano consultivo y asesor del Gobierno en materia de turismo.

Colombia no es ajena a la proyección mundial de la industria del turismo en cuanto al crecimiento en la movilidad de viajeros y, por ende, en la contribución al desarrollo de economías locales; prueba de ello ha sido el crecimiento paulatino de la llegada de viajeros a Colombia motivados por los productos y servicios que el país ofrece a través de Procolombia ${ }^{6}$. Todo esto, a pesar de los vaivenes

\footnotetext{
$6 \quad$ Procolombia es la entidad encargada de la promoción del turismo internacional, la inversión extranjera y las exportaciones no tradicionales en Colombia.
} 
políticos y sociales, y al deterioro de la imagen que ciertas situaciones internas han generado.

De otro lado se viene fortaleciendo la institucionalidad en materia turística: se posee un viceministerio de turismo que depende del Ministerio de Comercio, Industria y Turismo; ha crecido la legislación turística con tres leyes en menos de 16 años y el desarrollo de varias políticas conexas; asimismo se ha motivado la descentralización turística donde se han dado instrumentos jurídicos y de planificación para que los territorios desarrollen autónomamente proyectos tendientes a hacer del turismo una alternativa económica.

La Ley 300 de 1996 incluye el concepto de sostenibilidad en sus principios y esto por lo menos le da la oportunidad para que desde el espíritu de la norma se oriente el turismo hacia un desarrollo sostenible; igualmente relaciona el tema de la sostenibilidad con la competitividad, al formular los planes sectoriales de turismo.

Ya para la reforma de esta Ley con la 1558 de 2012 el concepto de sostenibilidad se evidencia más directamente desde sus principios, y marca desde el espíritu de la norma que esta actividad debe ir encaminada al desarrollo sostenible, cuando en el artículo 5 alude a la calidad y al cumplimiento de normas técnicas sectoriales en turismo sostenible (NTS-TS) para todos los prestadores de servicios turísticos: CONPES 3296 de 2004, Lineamientos para promover la participación privada en la prestación de servicios ecoturísticos en el Sistema de Parques Nacionales Naturales; CONPES 3397 de 2005, Política Sectorial de Turismo; CONPES 3658 de 2010, Lineamientos de Política para la recuperación de los Centros Históricos de Colombia.

El Consejo Nacional de Política Económica y Social (CONPES) es el máximo organismo de coordinación de la política económica en Colombia y es el que da orientación y línea de la política macro. De sus recomendaciones dependen programas y planes de desarrollo, los planes de inversión pública y los lineamientos del presupuesto general que cada año se presentan al Congreso de la República. Se encarga también de sembrar las bases para la protección de la industria nacional, otorgando certificados de desarrollo turístico y discutiendo los programas de apoyo y fomento al sector externo del país.

Con base en lo anterior es importante anotar que los CONPES 3296, 3397Política sectorial de turismo 2005 y 3658 , aparecen lineamientos asociados el concepto de sostenibilidad; esto marca un avance hacia el desarrollo sostenible del turismo que permite formular estrategias de desarrollo turístico y que puedan aplicarse a contextos locales y nacionales, concienciando a los diferentes actores sobre la sostenibilidad y generando un escenario de confianza, seguridad y 
calidad en el sector que se traduce en normas técnicas específicas, sellos de calidad, etiquetas ambientales, destinos sostenibles, etc., aspectos que directa o indirectamente permiten atraer mercados internacionales.

De los tres Conpes, el 3397 es el que brinda de una manera más explícita lineamientos importantes para el sector, partiendo de un diagnóstico general del mismo y centrando sus recomendaciones en el desarrollo de 6 estrategias, donde la sostenibilidad solo se refleja en la estrategia de la Preparación de la Oferta Turística y en el ítem que expresa literalmente: recuperación y sostenibilidad del patrimonio cultural y natural; aspectos que desarrolla planteando la importancia y complementariedad de los mismos para el desarrollo del turismo y la necesidad de salvaguardarlo. Las otras 5 estrategias reafirman los lineamientos de un desarrollo empresarial y competitivo del sector: 1. Fortalecimiento institucional necesario para el desarrollo de la actividad. 2. Mejoramiento de las condiciones de seguridad para los viajeros. 3. Establecimiento de una campaña agresiva y sostenida de promoción y mercadeo. 4. Formación del recurso humano y sensibilización turística. 5. Consolidación de un sistema de información turística que permita formular políticas de gobierno y el uso de tecnologías de la información y la comunicación.

Es en la década del año 2000 cuando comienzan a formularse políticas asociadas a las diferentes tipologías de turismo, que se enmarcan en la vocación y potencialidades de las regiones; estas abren el abanico y las bases conceptuales y metodológicas para abordar el turismo desde los criterios del desarrollo humano sostenible y de la sostenibilidad en general: Política para el Desarrollo del Ecoturismo, Política de Turismo Social: Hacia un Turismo accesible e incluyente para todos los colombianos, Política para el Turismo Cultural: Identidad y Desarrollo Competitivo del Patrimonio, Política de Turismo y Artesanías: Iniciativas conjuntas para el impulso y la promoción del patrimonio artesanal y el turismo colombiano, Documento de Política de Playas Turísticas: Lineamientos Sectoriales, Lineamientos de Políticas para el Desarrollo del Turismo Comunitario en Colombia y Política de Turismo de Naturaleza.

La política de ecoturismo recogió los lineamientos epistemológicos sobre el desarrollo, la biodiversidad, y la sostenibilidad; asimismo, los compromisos de los encuentros internacionales sobre el medio ambiente que marcaron las pautas para la construcción y desarrollo del turismo sostenible, en todas las actividades turísticas.

Varios de estos lineamientos se abordaron posteriormente en la formulación de las políticas para el Turismo Cultural y la Política de Turismo y Artesanías; veamos cómo se evidencia este aspecto en el objetivo general de la Política de Turismo Cultural: "Posicionar a Colombia, a partir de su diversidad cultural, 
como destino de turismo cultural interno y externo, a través del cual se generan dinámicas de desarrollo local sostenible, promoviendo la competitividad del patrimonio y la identidad de las regiones".

En el último lustro se formularon las políticas: Documento de Política de Playas Turísticas; Lineamientos de Políticas para el Desarrollo del Turismo Comunitario en Colombia y Política de Turismo de Naturaleza, las cuales visibilizaron de una manera más decisiva elementos de turismo sostenible en sus tres principales componentes: económico, social y ambiental. Un ejemplo de esto se evidencia en la política de turismo de naturaleza en su objetivo general: "Posicionar a Colombia como destino de turismo de naturaleza, reconocido por el desarrollo de productos y servicios altamente competitivos y sostenibles, que permitan preservar los recursos naturales y mejorar la calidad de vida de las comunidades receptoras".

En la carta de navegación para el turismo o plan sectorial que se formula por período de Gobierno cada cuatro años, el concepto de sostenibilidad aparece en los objetivos, en los fundamentos o en alguna estrategia a desarrollar. Veamos cómo se aborda en los tres últimos planes: Plan Sectorial 2006-2010: Colombia Destino Turístico de Clase Mundial; Plan Sectorial 2011-2014: Turismo Factor de Prosperidad para Colombia; Plan Sectorial 2014-2018: Turismo para la Construcción de la Paz.

Durante el Gobierno del presidente Álvaro Uribe Vélez se dio un impulso al turismo con el tema de inversión y seguridad democrática; adicionalmente, se dio gran aporte en la construcción de varios documentos políticos, dentro de los cuales el concepto de sostenibilidad fue visible y permitió generar estrategias y líneas de acción para su desarrollo; muestra de ello fue la política de ecoturismo que recogió los ejes de acción del desarrollo sostenible en el marco del contexto mundial.

En este Gobierno hubo avances significativos en el desarrollo del sector, pero también se visibilizaron problemáticas producto de los impactos inherentes a él. La campaña "Vive Colombia viaja por ella" permitió dar a conocer territorios que por problemas de orden público eran desconocido, se reactivó la economía de muchos pueblos por medio de la actividad turística, pero aquellos que no contaron con una planificación adecuada se vieron invadidos por esta actividad dejando impactos sobre todo en el aspecto ambiental. Finalmente es de destacar que durante este gobierno el turismo tuvo un gran impulso y permitió diseñar herramientas que todavía hoy están siendo interpretadas para un mejor uso.

En el gobierno actual, del presidente Juan Manuel Santos los planes sectoriales se enfocan hacia la competitividad de los destinos turísticos, en el marco 
de un desarrollo sostenible, aportando a la generación de empleo. Además, se está relacionando al turismo con la paz, siendo este el instrumento para mejorar la calidad de vida de las personas en un escenario de posacuerdo y posconflicto, desarrollando actividades alrededor del turismo de naturaleza y priorizando áreas de gran potencial turístico que otrora no se habían tenido en cuenta y que, a su vez, han sido impactadas por el conflicto tales como: Urabá, Caño Cristales, Valle del Sibundoy y sectores de la Sierra Nevada.

La estructura de los planes sectoriales tiene mucho en común y sus lineamientos se orientan a las dinámicas que el sector va exigiendo tanto en el ámbito nacional como en el internacional. En este sentido el gobernante de turno dirigirá su plan de gobierno, y el tema de sostenibilidad se incorpora para responder a directrices internacionales; no obstante, en muchos casos carece de profundidad y requiere que se incluyan líneas de acción propias para desarrollar el tema de sostenibilidad que les permitan a cada uno de los actores incorporarlas dentro de sus procesos.

Si bien esta normativa toca sin mucho desarrollo lo sostenible, son las Normas Técnicas Sectoriales en Turismo Sostenible para los prestadores de servicios turísticos las que comienzan a dar lineamientos más claros para su implementación. Estas se van socializando hasta el punto de pasar de ser de carácter voluntario a obligatorio para el 2017.

Es por ello que desde el 2007 y con el fin de atender la demanda cada vez más fuerte de los turistas por servicios de calidad y que no generen impactos, se diseñan estas normas enfocadas al turismo sostenible en algunos de los sectores del turismo: NTS-TS 001-1 Destinos Turísticos, 2014; NTS-TS 001-2 De Playas, 2015; NTS-TS 002 Establecimientos de Hospedaje, 2014; NTS-TS 003 Agencias de Viajes, 2007; NTS-TS 004 Gastronómicos y Bares, 2008; NTS-TS 006-1 Operadores de Eventos, 2012; NTS-S 006-2 Sedes para Eventos, 2012; NTS-TS 005 Transportes Especiales, 2009:

Las normas técnicas se asumen de carácter voluntario; sin embargo, algunos prestadores de servicios turísticos en pro de mejorar su servicio y, por ende, su competitividad, han realizado un proceso de certificación según la categoría a la que pertenecen, pero, muchas de estas normas, debido a presiones internacionales, se han convertido de obligatorio cumplimiento, como prerrequisito para la formalización del sector. Este hecho ha generado algunos impactos en los prestadores de servicios turísticos tales como:

1. Desconocimiento de la normativa y su respectiva ruta de aplicación, producto de la no existencia de unos canales de comunicación efectivos para la socialización de la norma. 
2. Aparecen y desaparecen resoluciones generando confusión en los prestadores de servicios turísticos, sin contar aquí con las dificultades que se presentan en la interpretación de la norma, debido a la falta de claridad de los responsables de dar respuesta a las inquietudes y, en muchos de los casos, con posiciones encontradas desde los entes gubernamentales.

3. Falta de acompañamiento permanente en todo el proceso de certificación, dejando solos a los prestadores de servicios turísticos en dichos procedimientos, lo que genera mayores lagunas y, por ende, deserción en los procesos de cualificación y certificación del sector, en casos como: qué se certifica, qué entidad lo hace, cuánto cuesta este proceso, qué beneficios concretos se obtienen, entre otros.

Con base en lo anterior han resultado muchas dudas en aspectos como: procedimientos para actualización de RNT (Registro Nacional de Turismo), requisitos de certificación en NTS (Normas Técnicas Sectoriales) para actualización de RNT y participación en convocatorias públicas para formular proyectos con el Estado y acceso a la banca. Un ejemplo de esto es la confusión presentada con la Resolución 2804 del 25 de junio de 2014 "Por el cual se reglamenta el cumplimiento de las normas técnicas de calidad expedidas por las Unidades Sectoriales de Normalización sobre Sostenibilidad Turística" y su posterior derogación en la Resolución 0148 del 19 de enero de 2015. "Por la cual se reglamenta el cumplimiento de las normas técnicas de calidad expedidas por las Unidades Sectoriales de Normalización sobre Sostenibilidad Turística". Se puede decir que en la formulación inicial no se dio una claridad en los procedimientos y en su aplicación, lo cual generó inconformidades en los prestadores de servicios turísticos llevando a un replanteamiento en los métodos de socialización de la norma.

De otro lado, no se ha dado el salto necesario hacia una política pública de sostenibilidad y de turismo sostenible, siendo más fácil una formulación de Normas Técnicas para empresas y personas que no alcanzan a impactar problemáticas mayores tales como: el futuro de los servicios ambientales, las declaraciones de áreas protegidas, los usos del suelo, la conservación y protección de áreas vulnerables, las concesiones hechas a multinacionales entre otras; aspectos que repercuten directamente en el desarrollo y sostenibilidad del turismo y que requieren medidas puntuales y estratégicas que estarían enmarcadas dentro de una política pública.?

7 En un informe de gestión publicado por el Ministerio de Comercio, Industria y Turismo, en el 2015, se identifican en términos de cifras las agencias de viajes, los establecimientos de alojamiento y hospedaje, los establecimientos gastronómicos y bares, los destinos turísticos de Colombia y los destinos de playas que se han certificado bajo NTS-TS. 
Para el sector estos requisitos, como se expresó antes, fueron de voluntario cumplimiento hasta el 2015, pero a partir del 2017 serán de obligatorio cumplimiento. Si bien estas Normas están orientadas al desarrollo de buenas prácticas en los componentes ambientales, socioculturales y económicos, no dan cuenta de la sostenibilidad del sector, y su adopción y aplicación pueden llevar a supuestos que alimentan la competencia desigual en los prestadores de servicios.

\section{Veamos:}

Un primer supuesto es pensar que el turista requiere de referentes asociados a procesos de certificación para su viaje. Un turista hoy hace uso de medios de comunicación que van desde referidos hasta redes y comunidades que están dando cuenta de las dinámicas de los actores de la cadena turística y, por ende, de los territorios, con un enfoque responsable y sostenible. En Colombia, por ejemplo tenemos dos destinos certificados en sostenibilidad (Puerto Nariño en Amazonas y Parque Arví en Medellín), un esfuerzo grande de toda la cadena para tener dicho reconocimiento, pero de poco impacto para el turista y aun para la misma población local, ya que a veces no hay ni siquiera conocimiento del mismo o no se refleja en la experiencia turística.

Un segundo supuesto es que la certificación logra ser una estrategia de comercialización efectiva. Es riesgoso pensar que esto sea obvio. El éxito de un sitio, atractivo o establecimiento depende de factores que tienen que ver con el servicio, las buenas prácticas y la innovación, y muchos se han ganado un reconocimiento de esta manera sin recurrir a sellos de calidad o procesos de certificación. Este supuesto puede traer consecuencias ya que puede asumirse como un negocio donde pueden beneficiarse grandes cadenas del turismo que "apoyan" emprendimientos locales y pueden pagar sin dificultades el costo de estos sellos.

Un tercer supuesto es pensar que estas certificaciones son el mejor camino para cambiar las prácticas económicas de las multinacionales del turismo (cadenas de alimentos, hoteles, transporte, agencias, etc.). Es de anotar que estas cadenas son las que más logran una certificación a corto plazo, debido a su estructura organizativa y financiera, y si realizan acciones visibles con las comunidades locales o con el medio ambiente, estas son paliativas a problemáticas mayores que a la luz del desarrollo sostenible y del turismo sostenible dejan mucho que desear.

Por dar solo un ejemplo, cadenas como McDonald's o cadenas de almacenes tienen líneas de comercio justo dentro de sus procesos de compras y comercialización, pero asfixian con las exigencias y políticas de pago a los pequeños proveedores; eso sin contar con los impactos que se generan con la compra de intangibles (manifestaciones culturales locales) por parte de las cadena hotele- 
ras para el recreo de turistas que "evidencian un gran apoyo a las poblaciones locales".

\section{Una mirada al desarrollo del turismo sostenible en Colombia}

A continuación se presentan algunos datos relevantes de lo que ha sido la evolución y desarrollo del turismo sostenible en Colombia.

En el Decreto 233 del 22 de abril de 1825, se comenzó a evidenciar un ordenamiento e identificación de beneficios en la cadena de servicios relacionados con uno de los componentes, el alojamiento, que posteriormente sería eje de la cadena productiva en el turismo. En este componente hubo una apuesta en lo social referente al servicio militar, y en lo económico en la exención de impuestos para los pequeños empresarios (posaderos), aspectos que, sin proponérselo, generaban las primeras bases de un desarrollo sostenible.

Es de anotar que en el Decreto 757 de 1972, en su artículo 7, se promueven lugares que por su interés cultural o belleza escénica pueden poseer un interés turístico, y se sugiere que dichos atractivos puedan tener una intensa actividad turística, lo cual en sí mismo no establece unos lineamientos que mitiguen impactos que contribuyan a una sostenibilidad. Se puede decir que desde esa época primó más una visión economicista del turismo, descuidando los factores sociales y ambientales.

Cabe mencionar que en los contextos que Colombia hace en la presentación de documentos relacionados con políticas resalta la importancia del crecimiento del sector al nivel mundial y su preocupación en la contribución del PIB que genera la actividad. Esta concepción no solo dista de una mirada integral de un turismo sostenible, sino que contribuye a socavar paulatina y silenciosamente el principal insumo de la actividad turística: el patrimonio cultural y natural de un destino. A continuación veamos parte de este esquema para dar continuidad a la reflexión:

Desde la década del 2000 hasta la fecha el turismo en Colombia ha mejorado de manera considerable, "Las llegadas internacionales mostraron un crecimiento sostenido, con una tasa de crecimiento promedio anual de 12 \%. En el 2011 el país recibió alrededor de 1,6 millones de visitas que generaron un ingreso de 2201 millones de dólares"8. Esto demuestra que el turismo es una actividad prometedora para Colombia, además de su evidente crecimiento al nivel mundial. En este mismo sentido la Organización Mundial del Turismo afirma que el turismo se ha convertido en la primera actividad económica de muchos países. Según las previsiones de la OMT, las llegadas de turistas internacionales a

8 Informe estadístico de La Comunidad Andina, de 2012, en la página 9. 
escala mundial crecerán un 3,3 \% anualmente entre 2010 y 2030, hasta alcanzar los 1800 millones de turistas.

Estas cifras, si bien son importantes para la medición de indicadores (aumento en número de turistas, cifras en dólares etc.) paradójicamente van asociadas a intervenciones significativas en los territorios, principalmente de infraestructura con unos costos de servicios ambientales altos. Prueba de ello es el incremento de la hotelería en las principales ciudades del país, sin una planificación clara en desarrollo vial y otros servicios de soporte, o el crecimiento del sector hotelero en zonas costeras que han sido íconos para el turismo de Sol y Playa, tales como Cartagena y Santa Marta. Ello sin contar con los intentos de desarrollar cadenas hoteleras dentro de zonas de áreas protegidas o en proyectos multimillonarios como Barú y otros ecosistemas costeros que no solo generan impactos ambientales sino socioculturales y económicos.

Dentro de las NTS-TS, se despliega un tratamiento especial en cada uno de los componentes de la sostenibilidad: lo ambiental, lo sociocultural y lo económico con su respectiva gestión de la sostenibilidad que, a la luz de lo anteriormente planteado, es desequilibrado. En materia de lo ambiental se consideran los aspectos de estrategias para el manejo responsable del agua, la energía, la contaminación auditiva, visual y atmosférica, los residuos sólidos, la conservación de la biodiversidad, el manejo de material impreso; en lo sociocultural, conservación del patrimonio tangible e intangible, la prevención de la explotación sexual y comercial de niños, niñas y adolescentes, y apoyo a campañas humanitarias; y en lo económico, contratación y generación de empleo, calidad y satisfacción del cliente, gestión de proveedores y capacitación a la comunidad.

Toda esta entelequia de buenas prácticas, si bien evidencia una ruta hacia un turismo sostenible no ayudan a resolver problemas estructurales que afectan directamente a la actividad turística en la vía de la sostenibilidad, por ejemplo, otorgamiento de licencias para la explotación minera; es importante resaltar que ya en los foros y encuentros regionales y nacionales de turismo el sector se viene pronunciando sobre este tema y su relación con el patrimonio y la biodiversidad: desplazamiento de poblaciones, producto de conflictos sociales donde priman intereses de multinacionales y el crimen organizado; elevado costo de vida y excesiva capacidad de carga en destinos turísticos posicionados tanto en el ámbito regional como en el nacional.

En el año 2006 se realizó otra reforma a la estructura del Ministerio de Comercio, Industria y Turismo, según Decreto 2785 de 2006. En esta nueva reforma se le asignó nuevas funciones al despacho del Ministro, tales como: "Ejercer la coordinación necesaria para fortalecer la competitividad y sostenibilidad del sector, de tal forma que el turismo encuentre condiciones favorables para 
su desarrollo en los ámbitos social, económico, cultural y ambiental", también se le dan nuevas funciones al despacho del Viceministro de Turismo entre las cuales se encuentra: "Concertar, ejecutar y evaluar la política turística, así como los planes y programas derivados de ésta, en articulación con las entidades competentes de los sectores privado y público del sector, con el fin de mejorar la competitividad y sostenibilidad de los productos y destinos turísticos y promover el turismo doméstico y receptivo".

Es importante mencionar que en muchas funciones y planteamientos de objetivos de la política, se encuentra la de mejorar la competitividad y sostenibilidad de los productos y destinos turísticos, aspecto que requiere de un tratamiento más amplio que contribuya a saldar esa relación de la competitividad con la sostenibilidad ya que en la misma definición de la competitividad turística está inserta de una manera muy clara la sostenibilidad, pero que desde el punto de vista de una economía de mercado riñen en su esencia. También en esta reforma se le asignan funciones a la Dirección de Calidad y Desarrollo Sostenible del Turismo y a la Dirección de Análisis Sectorial y promoción.

Dentro de estas funciones se resaltan las relacionadas con orientar procesos de calidad y certificación en el sector del turismo, así como encaminar a las regiones para la adopción e implementación de políticas en el territorio, pero es la función 7 la que tiene más pertinencia con la sostenibilidad: "Brindar asistencia técnica a los prestadores de servicios turísticos y a las entidades territoriales para la implementación de planes de excelencia turística y certificación de sostenibilidad".

En esta función es vago el concepto de excelencia turística: este comprende muchos aspectos que no necesariamente van ligados a la sostenibilidad; asimismo, toca el aspecto de la certificación en sostenibilidad. No es arriesgado decirlo, pero muchas de las funciones, y en particular de las que se hace mención, no son visibles en los territorios y se han circunscrito a tareas de capacitaciones puntuales sobre diseño de producto y planificación turística realizadas por funcionarios del viceministerio.

Otra de las dificultades que se presenta son las confusiones derivadas de la misma normativa donde no existen unos canales de interlocución claros con el ente gubernamental en materia turística para resolver las inquietudes de los prestadores de servicio. En otras palabras, la normativa no se baja a las regiones de acuerdo a los contextos, sino que su orientación padece del mal de otros sectores: el centralismo y por ende la burocracia, lo cual la hace legal, pero no legítima.

Si tomamos la Visión 2020 formulada desde el 2000 para el turismo colombiano, esta tampoco escapa de generalizaciones, y más aún no aparece de manera 
visible el concepto de sostenibilidad, propugnando más bien por una lógica de desarrollo empresarial diferenciadora y competitiva:

[...] Para el año 2020 Colombia habrá posicionado la actividad turística como una de las de mayor importancia para el desarrollo económico del país, generadora de empleo y eficiente redistribuidora de ingreso; por consiguiente, será un país que habrá fortalecido la competitividad de sus productos y destinos en un trabajo que permitirá una relación armónica nación-región, donde los sectores públicos nacional y local jugarán un papel determinante en la generación de condiciones óptimas para el desempeño empresarial (Visión 2020 en Plan Sectorial de turismo 2011-2014).

No obstante, en uno de sus apartes sí contempla la imperiosa necesidad de la participación de las comunidades en las dinámicas del desarrollo turístico, pero su enfoque es de un actor más en el territorio que hay que contar con él para que estas logren un beneficio económico. De igual forma los productos especializados que se plantean desarrollar como turismo cultural, de aventura, de salud, sol y playa, turismo de congresos, negocios e incentivos y sus respectivos subproductos necesitan plataformas de soporte y profesionalización que poco requieren para su concreción de un turismo comunitario:

[...] En el plano nacional, la unidad de planificación será el destino turístico; el país y los destinos habrán conseguido consolidar una cultura turística en la sociedad; el turismo habrá contribuido a preservar los valores sociales y patrimoniales y será un elemento fundamental para generar orgullo nacional. Las comunidades se habrán vinculado a la actividad turística, beneficiándose del desarrollo que proporciona esta industria. En tal sentido, se habrán consolidado diversas formas de gestión para la prestación de los servicios turísticos, poniendo especial énfasis en el turismo comunitario que permite que las empresas locales debidamente organizadas deriven beneficios de su participación en la cadena de valor del destino turístico (Visión 2020 Plan Sectorial de Turismo 2011-2014).

Asimismo, es clara en planteamientos como estos para su promoción internacional: "A nivel internacional Colombia se habrá concentrado en atraer turistas de altos ingresos, para lo cual habrá fortalecido su capacidad de investigación de mercados".

\section{Conclusiones}

Contar con una política integral en turismo sostenible podría ser el enlace que hace falta para que la política ambiental y turística encuentre elementos que brinden herramientas de aplicación a los diferentes actores tanto públicos como privados. Se puede decir que al nivel de gobernabilidad se cuentan con diversos lineamientos en ambas áreas ambiental y turística, pero cada una trabajando de manera independiente, cuando la realidad demuestra que en diversos proyectos siempre se requiere de ambas y es allí donde los agentes turísticos y ambientales no cuentan con elementos de articulación para hacer que tanto el turismo 
como la parte ambiental sean generadoras de conservación, protección, creando recursos que beneficien a comunidades donde se realicen ambas actividades. Por lo anterior se requiere una política clara y coherente que impulse acciones en todos los niveles del turismo para que sea sostenible.

Puede plantearse que el país ha concentrado sus esfuerzos en hacer del turismo una actividad que ocupe un lugar preponderante en la economía nacional; sin embargo, no ha tenido en cuenta las consecuencias de este posible desarrollo, como son los impactos ambientales, socioculturales y económicos. En otras palabras, el tema del desarrollo sostenible en general y del turismo sostenible en particular ha sido más un capítulo discursivo dentro de la estructura del turismo en Colombia y no una tarea a realizar.

Prueba de ello ha sido el crecimiento desaforado de la oferta hotelera en las principales ciudades que ha generado impactos en el aspecto urbanístico; el aumento de licencias mineras en zonas aledañas de áreas en proceso de conservación; el desplazamiento de poblaciones y el aumento del costo de vida en destinos turísticos, entre otras.

La formulación de muchas de las políticas carece de una agenda pública, lo que aumenta la problemática particular de cada subsector del turismo. Es fundamental que el Estado diseñe dicha agenda pública teniendo en cuenta cada uno de los actores que hacen parte de la actividad turística, ya que esto permitiría un panorama completo abarcando los temas ambientales, haciendo fundamental la inclusión de los conceptos y recomendaciones del turismo sostenible.

Puede decirse hoy que son las Normas Técnicas Sectoriales en Turismo Sostenible las que de una u otra manera orientan los temas de sostenibilidad en la actividad turística, aspectos que permiten competir en el mercado nacional e internacional; sin embargo, cuando una empresa turística desea aplicar NTS-TS debe solicitar asesoría para comprender todos los requerimientos y procedimientos que debe realizar, debido a su diversidad y complejidad; muchas de ellas no tienen o no solicitan el acompañamiento y prueba de ello son las pocas empresas prestadoras de servicios que hasta hoy están certificadas en el tema de sostenibilidad, en comparación con el gran número existente de prestadores de servicios turísticos y destinos turísticos.

Una de las constantes de la actividad turística en Colombia ha sido la informalidad y lo que se busca con las políticas, y normas existentes es que la actividad turística sea cada vez más formal y de calidad. La informalidad se ha debido a factores como: el turismo ha sido considerado como una actividad "de fácil emprendimiento", con poca estructura organizativa, sin muchos riesgos 
financieros y escasa formación; estos imaginarios no han beneficiado al sector lo que ha hecho más difícil la implementación de la normativa turística en general y más aún de la sostenibilidad en particular.

Llama la atención la forma como se inserta el concepto de sostenibilidad y su aplicación en las políticas de turismo: si bien se expresa claramente en los objetivos, no logra un desarrollo en el contenido de las mismas. Su construcción es un círculo vicioso que se centra en el deber ser de un desarrollo turístico que debería tener cualquier región del mundo: desarrollo de infraestructura, articulación de actores, diseño de productos especializados, fortalecimiento del talento humano, fomento y promoción, facilitación de la inversión extranjera, mejoramiento de la competitividad, incluyendo los procesos de calidad de los prestadores, de los productos y servicios. Pero dejando por fuera la sostenibilidad y lo que ello implica.

\section{Recomendaciones}

El panorama expuesto nos debe llevar a una reformulación de las políticas que rigen la actividad turística que van desde su misma estructuración, donde exista una participación real de todos los actores involucrados en la cadena y unos compromisos claros donde prime no solo la conservación del patrimonio natural y cultural del país, insumos necesarios de la actividad turística, sino una apuesta clara con el territorio donde el turismo sea una alternativa económica y participativa sin detrimento en la calidad de los productos y servicios.

Es importante que la construcción de los planes de desarrollo locales parta de las particularidades del territorio acompañado de un consenso con la comunidad, encaminándose hacia una construcción de política pública articulada a las líneas de país concentradas en las políticas, Conpes y planes sectoriales.

Es fundamental retomar los modelos que desde lo ambiental se han trabajado en materia de sostenibilidad y en particular el ejercicio realizado en las áreas protegidas de orden nacional, donde estrategias como la del ecoturismo comunitario ha impactado positivamente todos los elementos del sistema turístico: atractivos, infraestructura, planta turística, facilitaciones como elementos de la oferta y al turista como principal elemento de la demanda.

Estas estrategias parten inicialmente: del reconocimiento de comunidades ancestrales asentadas en los territorios de influencia de dichas áreas protegidas, delimitación de las áreas (zonas de amortiguación) desde donde se realizan conjuntamente acciones para la protección y conservación de dichos ecosistemas; capacitación en ecoturismo a dichas comunidades; promoción a la actividad artesanal buscando generar incentivos económicos y dinamización y recupera- 
ción de tradiciones culturales; manejo exhaustivo de agentes contaminantes que vayan en contra del recurso natural; manejo sostenible de los recursos de agua y energía y la exigencia de compromisos por parte de los visitantes y turistas para que hagan parte de dichos procesos sostenibles, y así minimizar y mitigar los impactos que el turismo pueda generar.

Si bien el modelo antes descrito se asocia directamente con las prácticas del ecoturismo, la sostenibilidad debe de ser transversal a todas las tipologías de turismo, incluyendo la de Sol y Playa que ha sido uno de los referentes más inmediatos del turismo masivo y depredador en todo el mundo.

\section{Referencias bibliográficas}

Acerenza, M. (2007) Desarrollo sostenible y gestión del turismo. Primera Edición. México: Ed. Trillas.

Brañes, R. (2000). El acceso a la justicia ambiental en América Latina: Derecho ambiental y desarrollo sostenible, ponencia publicada en Derecho ambiental y desarrollo sostenible. El acceso a la justicia ambiental en América Latina, Memoria del Simposio Judicial realizado en la ciudad de México del 26 al 28 de enero de 2000. México. Publicación de la Oficina Regional para América Latina y el Caribe y de la Procuraduría Federal de Protección al Ambiente, pp. 41 y 42.

Consejo Nacional de Política Económica y Social, Departamento Nacional de Planeación, Ministerio de Ambiente, Vivienda y Desarrollo Territorial, Unidad Administrativa Especial del Sistema de Parques Nacionales Naturales, Ministerio de Comercio, Industria y Turismo. (2004). CONPES 3296. Lineamientos para promover la participación privada en la prestación de servicios ecoturísticos en el Sistema de Parques Nacionales Naturales. Recuperado de https://colaboracion.dnp.gov.co/CDT/CONPES/ Econ\%C3\%B3micos/3296.pdf

Consejo Nacional de Política Económica y Social, Departamento Nacional de Planeación, Ministerio de Comercio, Industria y Turismo. (2005). CONPES 3397. Política Sectorial de Turismo. Recuperado de https://colaboracion.dnp.gov.co/CDT/Conpes/Econ\%C3\%B3micos/3397.pdf

Ministerio de Comercio, Industria y Turismo y Ministerio de Ambiente Vivienda y Desarrollo Territorial. (2003). Política para el desarrollo del Ecoturismo. Recuperado de http://www.fontur.com.co/aym document/aym_normatividad/2003/POLITICA_PARA_EL_DESARROLLO_DEL_ECOTURISMO.pdf

Ministerio de Comercio, Industria y Turismo, Artesanías de Colombia. (2009). Política de Turismo y Artesanías: iniciativas conjuntas para el impulso y la promoción del patrimonio artesanal y el turismo colombiano. Recuperado de http://www.mincit.gov.co/minturismo/loader.php?lServicio=Documento

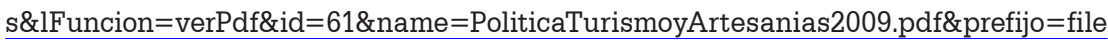

Ministerio de Comercio, Industria y Turismo, Departamento Nacional de Planeación. (2011). Plan sectorial de Turismo 2011-2014. "Turismo: factor de prosperidad para Colombia". Recuperado de http:// www.mincit.gov.co/minturismo/loader.php?1Servicio=Documentos\&lFuncion=verPdf\&id=40732\&na $\underline{\mathrm{me}=\text { PlanSectorialTurismo2011.pdf\&prefijo }=\text { file }}$

Ministerio de Comercio, Industria y Turismo, Ministerio de Cultura. (2007). Política de Turismo Cultural: Identidad y desarrollo competitivo del patrimonio. Recuperado de http://www.mincit.gov.co/ minturismo/loader.php?1Servicio=Documentos\&lFuncion=verPdf\&id=69\&name=PoliticaTurismoCu ltural.pdf\&prefijo $=$ file 
Ministerio de Comercio, Industria y Turismo. (2009). Política de Turismo Social: Hacia un turismo accesible e incluyente para todos los colombianos. Recuperado de http://www.mincit.gov.co/minturismo/ loader.php?1Servicio=Documentos\&lFuncion=verPdf\&id=62\&name=TurismoSocial.pdf\&prefijo=file

Ministerio de Comercio, Industria y Turismo. (2011). Documento de Política de playas turísticas: Lineamientos sectoriales. Recuperado de http://www.mincit.gov.co/minturismo/loader.php?lServic$\underline{\text { io }=\text { Documentos\&lFuncion }=\text { verPdf\&id }=62473 \& \text { name }=\text { politica-playas-turisticas } . \text { pdf\&prefijo }=\text { file }}$

Ministerio de Comercio, Industria y Turismo. (2012). La Ley 1558, por la cual se modifica la Ley 300 de 1996-Ley General de Turismo, la Ley 1101 de 2006 y se dictan otras disposiciones. Recuperado de http:// www.secretariasenado.gov.co/senado/basedoc/ley_ 1558 _2012.html

Ministerio de Comercio, Industria y Turismo. (2012). Lineamientos de políticas para el desarrollo del turismo comunitario en Colombia. Recuperado de http://www.citur.gov.co/upload/publications/documentos/84.Politica_de_Turismo_Comunitario.Lineamientos_de_politica.pdf

Ministerio de Comercio, Industria y Turismo. (2012). Política de Turismo de Naturaleza. Recuperado de http://www.mincit.gov.co/loader.php?1Servicio=Documentos\&lFuncion=verPdf\&id=64224\&name $=\mathrm{P}$ OLITICA_DE_TURISMO_DE_NATURLAEZA_22_SEPTIEMBRE.pdf\&prefijo=file

Ministerio de Desarrollo Económico. (1996). Ley 300 de 1996, Ley General de Turismo. Recuperado de http://www.alcaldiabogota.gov.co/sisjur/normas/Norma1.jsp?i=8634 
